\title{
HOMOGENEOUS SPHERE BUNDLES AND THE ISOTROPIC RIEMANN MANIFOLDS
}

\author{
TADASHI NAGANO
}

\section{Introduction}

Let $M$ be a connected metric space and $H$ an isometry group of $M$ which leaves fixed a point $p$ in $M . \quad M$ is said $H \cdot i$ sotropic at $p$ when, for any two points $q$ and $r$ of $M$ at the same distance from $p$, there exists an isometry in $H$ which carries $g$ to $r$. When $H$ coincides with the maximum isometry group leaving $p$ fixed, $M$ is said merely isotropic at $p$.

Assuming further that $M$ is compact and the isometry group of $M$ is transitive, H. C. Wang [26] proved that $M$ is a homogeneous space of a compact Lie group and that the homogeneous space is one of the spaces: a sphere, a (real, complex or quaternion) projective space and the Cayley projective plane.

J. Tits [25] and H. Freudenthal [11] succeeded in determining the homogeneous space $M$ under weaker conditions. Here are Freudenthal's hypotheses on $M$, which are more general than those of Tits. $M$ is a connected locally compact Hausdorff space. $M$ admits a transitive group $I$ of topological trans. formations having the properties $(S),(V)$ and $(Z)$.

( $S$ ): Given a compact subset $A$ and a closed subset $B$ of $M$ with $A \cap B$ $=\phi$ (the empty set), there exists an open set $U \neq \phi$ such that for any $\tau$ in $I$, $\tau(U) \cap A \neq \phi$ implies $\tau(U) \cap B=\phi$.

By $(S), M$ has an $I$-invariant uniformity, from which he defines a uniformity on $I$ so that $I$ is a topological group.

$\left(V^{r}\right): I$ is complete.

$(Z)$ : $J$ denoting the isotropy subgroup of $I$ at a point $p$, there exists an orbit $J(q), q \subseteq M$, such that $M-J(q)$ is not connected.

$J$ is compact. Using Yamabe's theorem, Freudenthal showed that $I$ is a Lie group, and so $M$ admits an $I$-invariant Riemannian metric. $M$ is then $J$ isotropic at $p$. He determined $I, J$ and $M=I / J$ by studying the Lie algebras 
of $I$ and $J$.

On the other hand $\mathrm{R}$. Bott [6] proved that, if all the geodesics of a Riemannian manifold $M, 2 \leqq \operatorname{dim} M$, are simply closed and of the same length, then $M$ is compact and two cases are possible: 1) the fundamental group of $M$ is of order two and the universal covering of $M$ is a homological sphere, or 2) $M$ is simply connected and its Poincaré polynomial over any field is of the form $1+t^{k+1}+t^{2(k+1)}+\cdots+t^{(m+1)(k+1)}$.

For the proof he used the Morse theory and theory of spectral sequences.

Now our main theorem states:

TheOREM 1. Let $M$ be a connected manifold with a Riemannian metric of class $C^{2}$. If $M$ is isotropic at a point $p$, then there exists $a C^{1}$-diffeomorphism $\delta$ of $M$ onto one of the spaces: a euclidean space, a sphere, a real elliptic space, a complex elliptic space, a quaternion elliptic space and the Cayley elliptic plane. Further $\delta$ induces an isomorphism of the isotropy group $J$ at $p$ into the isotropy group at $\delta(p)$, where the euclidean space and the sphere are given the usual metrics, and the elliptic spaces are the projective spaces with the metrics of irreducible symmetric spaces of rank 1 ; i.e. the last three Riemannian homogeneous spaces are $U(m+1) /(U(1) \times U(m)), S p(m+1) /(S p(1) \times S p(m))$, and $F_{4} /$ Spin $(9)$. (See [8] for these notations but Cartan's notation $F_{4}$.)

The condition to be "isotropic at $p$ " can be replaced by a seemingly weaker one: the linear isotropy group induced from $J$ on the tangent space at $p$ is transitive on the unit sphere, according to the preceding paper [21]. The assumption of Theorem 1 is equivalent to the condition: $M$ is a connected paracompact differentiable manifold of class $C^{3}$ on which a compact Lie transformation group admits an $(n-1)$-dimensional orbit and a fixed point, $n$ being the dimension of $M$ (Corollary 1.1).

When $M$ is not compact Theorem 1 follows from results in [21], which will be summarized in Section 1 . When $M$ is compact, $F$ shall denote the set of the farthest points from $p$. The assumption gives that $F$ is a $J$-orbit. $M-\{p\}$ will turn out to have a differentiable fiber bundle structure over $F$. The bundle is determined by some subbundle $S . \quad S$ is a homogeneous spherebundle (whose bundle space $S$ is) homeomorphic to a sphere, where, unlike certain other authors, we mean by a homogeneous bundle a fibre bundle on which a connected Lie group of bundle automorphisms operates transitively. 
Based on this fact we shall investigate topological properties of $F$ in Sections 2 and 3. In particular the Poincaré polynomial of $F$ will be found in the form: $P(t ; F)=1+t^{k+1}+\cdots+t^{m(k+1)}, k$ denoting the dimension of the fibre of $S$. It will further turn out that $k$ is odd and the Euler characteristic $\chi(F)$ of the homogeneous space $F$ is positive. The last property is important for the determination of $F$.

We shall next determine all homogeneous sphere-bundles homeomorphic to spheres. The associated principal bundle of any such bundle is a Lie group $G$ fibered by a closed subgroup $H$. Not only $G$ but $H$ is transitive on a sphere. The local structures of such groups were enumerated by $D$. MontgomeryH. Samelson [18] and A. Borel [1]. In Section 4 we shall determine the local structures of $G$ and $H$. Their global structures and the operation of $H$ on the fibre $S^{k}$, therefore the bundle $S$, will be determined in Section 6 (Theorem 3 ). For this purpose we need the following theorem.

THEOREM 2. Let $H$ be a compact Lie group which operates effectively and transitively on a simply connected homological sphere $S$ of dimension $n-1, H^{\prime \prime}$ be the identity component of $H$, and $C$ be the centralizer of $H$ in the group of topological transformations of $S$. Moreover let $H^{\prime}$ be a closed connected subgroup of the orthogonal group $O(n)$ such that $H^{\prime}$ is transitive on the unit sphere $S^{n-1}$ and locally isomorphic to $H$. Then there exists a homeomorphism of $S$ onto $S^{n-1}$ which induces an isomorphism of $H \cdot C$ into $O(n)$ carrying $H^{0}$ onto $H^{\prime}$.

Given $H$, there always exists the group $H^{\prime}$ ([18], [1]). A part of this theorem was announced by A. Borel without a full proof [3] (See also his review on [17] in Zentralblatt Math. 42, p. 259). A similar result was obtained by Y. Matsushima [17] in the case of homological sphere over rationals with a finite number of exceptions for $H^{0}$. Our theorem implies that for any transitive group $H^{\prime}$ locally isomorphic to $H^{0}$ there exists an isomorphism of $H^{0}$ onto $H^{\prime}$ carrying the isotropy subgroup of $H^{0}$ onto that of $H^{\prime}$; i.e. the operations of compact connected groups transitive on the sphere are determined by their local structures.

In Section 5 we shall prove the part of Theorem 2 which will be used in Section 6 to prove Theorem 3. By this theorem the bundle $S$ is all given and so is the bundle $M-\{p\} ; M-\{p\}$ will turn out to be equivalent to 
$M^{\prime}-\left\{p^{\prime}\right\}, M^{\prime}$ denoting one of the spaces mentioned in Theorem 1 . In particular there exists a diffeomorphism $\delta$ of $M-\{p\}$ onto $M^{\prime}-\left\{p^{\prime}\right\}$.

In Section 7 we shall complete the proof of Theorem 2 in order to show that $\delta$ extends to $M$ and that $\delta$ induces an isomorphism of the isotropy group $J$. Theorem 1 will thereby be established in 7 .

In Section 8 we shall determine the Riemannian metric (and the isometry group) of $M$ under the assumption of Theorem 1 added to the hypothesis that $M$ is Riemannian homogeneous. This gives another proof of the above mentioned result of Wang, Tits and Freudenthal under the assumption that the isometry group is a Lie group. Thanks to the topological study of $M$ and $F$ in the non-homogeneous case, the proof is very simple.

Differentiability class. Since geodesics on $M$ play an essential role, $M$ must be of differentiability class $C^{3}$. If one formulates P. S. Mostert's theorem [20] in the form shown in Section 1, one will be able to generalize Theorem 1 to the case where $M$ is a topological manifold, the map $\delta$ in the conclusion being then not a diffeomorphism but a homeomorphism. That $M$ is a topological manifold one cannot derive from Freudenthal's hypotheses except transitivity of $I$.

In Appendix we shall prove an unpublished theorem of Matsushima which will be used in Section 8 .

The author wishes to express his hearty thanks to Professor Kentaro Yano for his instruction and encouragements. He feels deeply grateful to his friend A. Hattori for his aids given to the author to surmount several obstacles. Further he thankfully acknowledges many valuable suggestions given by Professor N. Iwahori and his friends M. Ise, M. Sugiura and I. Tamura. Professor Iwahori kindly read the manuscript.

\section{Preliminaries}

In this section we summarize the results obtained in the preceding paper [21] which we shall need in the sequel.

Let $N$ be a connected non-compact differentiable manifold with a Riemannian metric of class $C^{2}$, and $G$ be a compact connected isometry group of $N$. $n$ shall denote the dimension of $N$. Throughout this section we shall preserve the assumption : 
(1.1) There exists an $(n-1)$-dimensional $G$-orbit and $a G$-orbit $B=G(b), b \in B$, such that the complement $N-B$ is connected.

By a perpendicular to $B$ (ending at $r \in B$ ) we mean a geodesic ending at a point $r$ of $B$ and orthogonal to $B$ at $r$.

(1.2) Given a point $q$ in $N$, there exists a unique perpendicular to $B$ which starts at $q$.

(1.3) Given $a$ point $b$ in $B$, the union $E$ of all the perpendiculars to $B$ ending at $b$ is a submanifold on which a normal coordinate system with.center $b$ is valid all over. Furtheremore $E$ is $H$-isotropic at $b$ where $H$ is the isotropy subgroup of G al b.

In particular $E$ is $C^{1}$-diffeomorphic with a euclidean space. $H$ is a linear group on $E$ in terms of the normal coordinates.

From (1.2) it follows that

(1.4) $N$ has a (differentiable) fibre bundle structure over $B$ associated uith $(G, G / H, H)$, i.e. $G$ fibered by $H$.

From (1.3) and (1.4) follows

(1.5) For an arbitrary point $q$ in $N-B$, the orbit $G(q)$ is of dimension $n-1$ and has a sphere-bundle structure associated with $(G, G / H, H)$. A sphere is understood to consist of two points when it is zero-dimensional. This bundle is a subbundle of $N$.

(1.6) The manifold $N$ and the bundle structure on $N$ is determined uniquely by the subbundle $G(q)$ and the transformation group $G$ in the sense that if (1) $G$ operates also on another non-compact Riemann manifold $N^{\prime}$ satisfying (1.1), (2) $H$ is the isotropy subgroup at a point $b^{\prime}$ of $B^{\prime} \subset N^{\prime}$, and (3) there exists a diffeomorphism of the sphere $H(q)$ onto the sphere $H\left(q^{\prime}\right), q^{\prime} \in N^{\prime}-B^{\prime}$, which induces an automorphism of $H$, then $N$ is diffeomorphic to $N^{\prime}$ and the bundle structures on $N$ and $N^{\prime}$ are equivalent. Eventually

(1.7) The diffeomorphism of $N$ onto $N^{\prime}$ can be so chosen that each perpendicular to $B$ is mapped to a perpendicular to $B^{\prime}$.

\section{The subspace $F$ of $M$}

In this section we preserve the hypothesis of Theorem 1 . 
We may assume that the dimension $n$ of $M$ is greater than 1 , because otherwise Theorem 1 is clear. $M-\{p\}$ is then connected. By a well known theorem of Dantzig-Waerden, the maximum isometric isotropy group $J$ at $p$ of $M$ is compact. For a small number $e$ the totality $S$ of the points of $M$ at the distance $e$ from $p$ is homeomorphic to the $(n-1)$-sphere. Since $J$ is transitive on $S$, so is the identity component $J^{0}$. A simple connected and compact subgroup $G$ is therefore transitive on $S$ by a theorem of Montgomery and Samelson [18].

\section{(2.1) Theorem 1 is true when $M$ is not compact.}

When $M$ is not compact, we put $M=N,\{p\}=B, J^{0}=G$. (1.1) holds good and we have (1.4). The base space $B$ being a point, the bundle space $M$ coincides with the fibre $E$. By (1.3), a normal coordinate system $\nu$ with center $p$ is valid on $M . \quad \nu$ induces an isomorphism of $J$ onto the linear isotropy group which can be considered as a subgroup of $O(n)=$ the group of all linear transformations preserving the quadratic form on $T$ defined by the Riemannian metric tensor.

$M$ has a metric $d$ defined by the Riemannian metric. Denote by $F$ the set of all the farthest points from $p$. The distance function $d(q)=d(p, q)$ then attains its maximum value on $F$.

(2.2) $F$ is a compact and connected submanifold with $0 \leqq \operatorname{dim} F<n$.

Since $F$ is a $J^{0}$-orbit, $F$ is a compact connected submanifold. $M$ being compact, $F$ is not empty. Finally, if $\operatorname{dim} F=n, F$ is open and closed, which leads to a contradiction $F=M$.

\section{(2.3) $M-F$ is connected.}

$M$ being compact, any point $q$ in $M$ can be joined to $p$ by a geodesic $\gamma(q)$ of arc length equal to $d(p, q)$. If $q$ belongs to $M-F$, then $\gamma(q)$ contains no points of $F$ by the definition of $F$. Hence $M-F$ is connected.

(2.4) $S$ is a homogeneous sphere-bundle over $F$ with bundle space homeomorphic to the $(n-1)$-sphere. (For the definition of a homogeneous bundle, see the introduction.)

Putting $M-\{p\}=N, F=B, J^{0}=G$, we find the condition (1.1) satisfied due to (2.3). Hence (1.5) applies. Each element of $G$ is clearly a bundle-automorphism. 
(2.5). In case $2<n$, the fundamental group $\pi_{1}(M)$ of $M$ is isomorphic to $\pi_{1}(F)$. In fact we have $\pi_{1}(M)=\pi_{1}(M-\{p\})$ because the fundamental group is determined by the 2 -skeleton. From (1.3), (1.4) and the proof of (2.4), we deduce that $M-\{p\}$ is a fibre bundle over $F$ with fibre homeomorphic to a euclidean space. Hence we have $\pi_{1}(M-\{p\})=\pi_{1}(F)$.

\section{Topological properties of $F$}

Some of the facts proved in this section are known. Throughout this section we assume:

(3.0) $S$ is a sphere-bundle whose bundle space, denoted also by $S$, is a simply connected $(n-1)$-dimensional homological sphere. The dimension of the base $F$ satisfies the inequality $0<\operatorname{dim} F<n-1$. A homological sphere is by definition a compact space whose integral homology group is isomorphic with that of a sphere.

Let $k$ be the dimension of the fibre. We have $0<k$.

(3.1) F is simply connected, because $S$ is simply connected and the fibre is connected.

(3.2) The $i$-th homotopy group $\pi_{i}(F)=0$, if $i-1<k$ or if $k=1$ and $2<i<n-1$.

We have $\pi_{i}(S)=0$ for $i<n-1$ by Hurewicz' theorem and (3.0). Now (3.2) follows from the exact sequence ([24] p. 91):

$$
\cdots \rightarrow \pi_{i}(S) \rightarrow \pi_{i}(F) \rightarrow \pi_{i-1}\left(S^{k}\right) \rightarrow \pi_{i-1}(S) \rightarrow \cdots \cdot
$$

By (3.0) and (3.1) we obtain Gysin's sequence ([23] p. 470):

$$
\cdots \rightarrow H^{i}(F) \rightarrow H^{i}(S) \rightarrow H^{i-k}(F) \rightarrow H^{i+1}(F) \rightarrow \cdots,
$$

where $H^{i}(X)$ is the $i$-th cohomology group of $X$ over the integers. We thus have

$$
H^{i-k}(F)=H^{i+1}(F) \quad(0<i<n-2) .
$$

Combining this with (3.2), we get from Hurewicz' theorem

(3.4) F is free of torsion and has the Poincaré polynomial of the form:

$$
P(t ; F)=1+t^{k+1}+\cdots+t^{m(k+1)}, \quad m(k+1)=\operatorname{dim} F .
$$

(3.5) The number $n-1$ is odd. 
If $n-1$ is even, Leray's theorem ([15] P. 190) giving the relation the Euler characteristic $\chi(S)=\chi\left(S^{k}\right) \chi(F)$

implies that $k$ is even, contrary to the following (3.6).

(3.6) The number $k$ is odd.

The isomorphism in (3.3) is given by

$$
h: x \in H^{i-k}(F) \rightarrow x \cdot \Omega \in H^{i+1}(F),
$$

where $\Omega$ is the $(k+1)$-dimensional characteristic class of Whitney. If $k$ is even, $2 \Omega$ is known to vanish. Then $h$ cannot be an isomorphism of $H^{0}(F)=Z$ onto $H^{k+1}(F)$.

(3.7) The Euler characteristic $\chi(F)$ of $F$ equals $(\operatorname{dim} F) /(k+1)+1$; hence it is positive.

From (3.4) and (3.6) we find $\chi(F)=P(-1 ; F)=m+1>0$. Finally (3.4) gives

(3.8) $\quad k<\operatorname{dim} F$.

\section{The homogeneous space $F$}

We preserve the hypothesis (3.0) and the notation $k$ throughout this section. Further we assume that $S$ is a homogeneous bundle. Since $S$ is compact and simply connected there exists a compact and connected group of automorphisms which is transitive on $S$ (Montgomery-Zippin [19] p. 226). S being a simply connected homological sphere, a theorem of Montgomery and Samelson (See the proof of Theorem $1^{\prime}$ in [18]) tells us that

(4.1) Some simple connected and compact Lie group $G$ of automorphisms is transitive on $\mathrm{S}$.

$G$ is considered to be effective on $S$. Denote by $H$ the subgroup of $G$ which leaves invariant a fibre $S^{k}$. We have $G / H=F$.

(4.2) The bundle $S$ is associated with the principal bundle $(G, G / H, H)$. The structure group $H$ is transitive on the fibre $S^{k}$ of $S$.

(4.3) $\pi_{i}(H)=\pi_{i}(G)$, if $0<i<k$ or if $k=1$ and $2<i<n-2$.

This follows from (3.2) and the exact sequence:

$$
\cdots \rightarrow \pi_{i+1}(F) \rightarrow \pi_{i}(H) \rightarrow \pi_{i}(G) \rightarrow \pi_{i}(F) \rightarrow \cdots .
$$


(4.4) If $1<k, H$ is semi-simple.

Since $G$ is semi-simple (because $\operatorname{dim} S>1$ ), $\pi_{1}(G)$ is finite. Hence (4.3) implies (4.4). (As regards literatures on the topology of Lie groups we refer the reader to [22] and [4].)

(4.5) If $4<k, H$ is simple.

In fact we have $\pi_{3}(H)=\pi_{3}(G)=Z$.

(4.6) The rank of $G$ equals that of $H$.

This is a consequence of (3.7) and a theorem of Hopf and Samelson.

(4.7) A compact connected and simple transformation group operating on $a(2 r-1)$-sphere is $D_{r}, r>2 ; A_{r-1} ; C_{r / 2}, r=$ even; or $B_{r / 4+2}, \quad r=4$ or 8 . (These notations of Cartan, $A, B, C, D$ concern the local structures of groups only.)

See D. Montgomery-H. Samelson [18] and A. Borel [1], or H. Freudenthal [11]. (Later in Section 5 we shall give a brief sketch of a proof.)

(4.8) $\pi_{5}\left(D_{r+1}\right)=\pi_{5}\left(B_{r / 1+2}\right)=0, \pi_{5}\left(A_{r-1}\right)=Z, \quad \pi_{5}\left(C_{r / 2}\right)=Z_{2}, \quad \pi_{5}\left(B_{2}\right)=Z_{9}, \quad \pi_{5}\left(D_{2}\right)$ $=Z_{2}+Z_{2}, \pi_{5}\left(D_{3}\right)=Z$, where $r$ is greater than 2 and $Z_{2}$ is the group of order 2.

These relations are found in Bott's table [5], except the last three [24].

(4.9) If $5<k$, then $k=7$ and $G$ is $B_{4}$ and $H$ is $D_{1}$.

With $G, H$ is simple by (4.5). Hence $H$ is almost effective on $S^{k}$ by (4.2). On account of (3.5) and (3.6) together with (4.1) and (4.2), we can apply (4.7) to $G$ and $H$. On the other hand $H$ must be $D_{3}$ or $D_{4}$ by $(4.3),(4.6),(4.8)$ and the inequality $\operatorname{dim} G-\operatorname{dim} H>0 . \quad D_{3}$ must be excluded because of (4.7) and the inequality.

(4.10) $5 \neq k$.

Assume $5=k . \quad H$ is simple by (4.5). $H$ is $D_{3}$ or $A_{2}$ by (4.7). Hence the rank of $G$ is 3 or $2 . n-1$ is not less than 11 by (3.8). Therefore $G$ is $C_{3}$. It is known ([24] p. 132) that $\pi_{4}\left(C_{3}\right)=Z_{2}$, while $\pi_{1}\left(D_{3}\right)=\pi_{4}\left(A_{2}\right)=0$, contrary to (4.3).

In order to investigate the remaining cases $k=3$ and $k=1$ we need some other known facts. Denoting the Poincare polynomial (over the real field) of $G$ and $H$ by $\Pi_{i}\left(1+t^{s_{i}-1}\right)$ and $\Pi_{\imath}\left(1+t^{r_{i}-1}\right)$ respectively, we assert:

$$
\left(1-t^{(m+1)(k+1)}\right) \Pi\left(1-t^{r_{i}}\right)=\left(1-t^{k+1}\right) \Pi\left(1-t^{s_{i}}\right), 0<m .
$$


This follows from (3.1), (3.4), (4.6) and Leray's theorem ([2] p. 191):

(4.11') If $H$ is a closed connested subgroup of a compact connected Lie group $G$ and if $H$ has the same rank as $G$, then the Poincaré polynomial $P(G / H ; t)$ of $G / H$ equals $\Pi\left(1-t^{s_{i}}\right) / \Pi\left(1-t^{r_{i}}\right)$.

(4.12) Let $L_{\delta}$ be a proper closed subgroup of a compact simple group $\delta_{r}(\delta=A$, $B, C, D)$ such that

1) $\operatorname{rank} L_{\delta}=\operatorname{rank} \delta_{r}=r$,

2) the center of $L_{\delta}$ is of dimension $\leqq 1$,

3) $L_{\delta}$ is simple or a local product of two simple groups.

Then locally

$L_{A}$ is $T \times A_{r-1}$;

$L_{B}$ is $B_{1} \times B_{1}(r=2), D_{r} \times B_{r-j}(2<j \leqq r), D_{j} \times D_{r-j}(2<j<r-2)$,

$T \times A_{r-1}, T \times D_{r-1}$ or $T \times B_{r-1}$;

$L_{C}$ is $C_{j} \times C_{r-j}(0<j<r), T \times A_{r-1}$, or $T \times B_{r-1}$;

$L_{D}$ is $D_{j} \times D_{r-j}(2<j<r-1), T \times A_{r-1}, T \times D_{r-1}$,

where $T$ is the one-dimensional toral group. (See Theorem II in Wang [27]).

(4.13) In case $k=3, G$ is $C_{n / 4}$ and $H$ is $C_{1} \times C_{n / 4-1}(8 \leqq n)$.

Since $G$ is simple, the coefficients of $t^{2}$ and $t^{4}$ in (4.11) equal 0 and -2 respectively. The number $(m+1)(k+1)$ being greater than 4 , it follows that $H$ is semi-simple and a local product of two simple subgroups. One of them is of rank 1 because $H$ is transitive on $S^{3}$. Inspecting the tables in (4.12) and (4.7), one finds that

1) $G$ is $C_{n / 4}$ and $H$ is $C_{1} \times C_{n / 4-1}$, or

2) $G$ is $B_{4}$ and $H$ is $D_{3} \times B_{1}$.

But the case 2) does not occur, as we find by substituting the known Betti numbers of $G$ and $H$ into (4.11) or by calculating the dimensions:

$$
\begin{aligned}
& \operatorname{dim} B_{4}-\operatorname{dim}\left(D_{3} \times B_{1}\right)=18, \text { while } \\
& \operatorname{dim} G-\operatorname{dim} H=\operatorname{dim} F=(n-1)-k=15-3 .
\end{aligned}
$$

Wang [27] proved that

(4.14) If a compact connected Lie group $G$ has the same rank as $H$ and if the 
factor space $F=G / H$ is simply-connected, then the homogeneous space $F$ is determined by the local structure of $G$ and $H$, i.e. if $G$ and another analogous group $G^{\prime}$ operate effectively on $F$ and $F^{\prime}=G^{\prime} / H^{\prime}$ (which is also simply-connected) respectively and if $G$ and $H$ are locally isomorphic onto $G^{\prime}$ and $H^{\prime}$ respectively, then there exists an isomorphism $\alpha$ of $G$ onto $G^{\prime}$ carrying $H$ onto $H^{\prime}$.

(4.15) If $k=1$, three cases are possible:

1) $G$ is $A_{1}$ and $H$ is $T$,

2) $G$ is $A_{n / 2-1}$ and $H$ is $T \times A_{n / 2-2}$, and

3) $G$ is $C_{n / 1}$ and $H$ is $T \times C_{n / 4-1}$.

$F$ is thus diffeomorphic to a complex projective space.

By (4.11), the center of $H$ is one-dimensional. When $H$ is $T, G$ is $A_{1}$ by (4.11) or (4.6), and we obtain the case 1 ). When $H$ is not $T, H$ is a local product of $T$ and a simple semi-simple group. Hence (4.12) applies to $H$. As in the preceding cases, we get the possibilites 2 ), 3) and

4) $G$ is $B_{4}$ and $H$ is $T \times B_{3}$

by using (4.7), Lemma 4 in [18] (if $n-1=5$ ), (4.3) (if $n-1>5$ ) and (4.8). The same results are obtained with another method. The Euler characteristics of the spaces $\delta_{r} / L_{\delta}$ defined by $L_{o}=\left(T \times \delta_{r-1}^{\prime}\right)$ in (4.12) in that order are $r+1$, $2^{r}, 4 r, 2 r, 2^{r}, 2 r, 2^{r+1}$ and $2 r$, owing to Theorem VII in Wang [27]. On the other hand, if the rank of $G$ equals $r$, we see from (3.7) and (4.7) that the Euler characteristic of $F$ is $r+1,4 r-8,2 r$ or $r$ according as $G$ is $A_{r}, B_{r}, C_{r}$ or $D_{r}$. Comparing these values of the Euler characteristic calculated in two ways, we have 2), 3) and 4) again. In case 4$),(4.14)$ shows that $F$ is the Grassmann manifold $S O(9) /(S O(2) \times S O(7))$. (For the notations $S O$, $S U$, etc., see [8]). Applying C. Ehresmann's method [7], we find that the 3rd torsion group of $F$ is not trivial, contrary to (3.4). Now only 2) and 3) remain. In case 3), $G$ is mapped into $S U(n / 2)$ by a natural isomorphism ([8] p. 21), which sends $H$ to the isotropic subgroup $U(1) \times S U(n / 2-1)$. Hence $F$ is diffeomorphic to the complex projective space $S U(n / 2) /(U(1) \times S U(n / 2-1))$.

Summarizing the above results, we get

(4.16) Let $F$ be the base space of a homogeneous sphere-bundle $S$ which is a homological spluere. If $\operatorname{dim} S=n-1,0<\operatorname{dim} F<n-1$ and $S$ is simply 
connected, then as a homogeneous space $F$ is isonzorphic to (1) the 8-sphere $\mathrm{SO}(9) / \mathrm{SO}(8),(2)$ the quaternion projective space $\mathrm{Sp}(n / 4) /(\mathrm{Sp}(1) \times \mathrm{Sp}(n / 4-1))$, $8<n$, (3) the 4-sphere $S p(2) /(S p(1) \times S p(1))$, (4) the 2-sphere $S O(3) / S O(2)$, and (5) the complex projective space $S U(n / 2) /(U(1) \times S U(n / 2-1))$.

These spaces correspond to $(4.9),(4.13)(8<n),(4.13)(8=n),(4.15 ; 1)$, and $(4.15 ; 2)$. See $(4.14)$ also.

\section{The homogeneous homological spheres}

In this section we shall establish a part of Theorem 2 (in the introduction) : (5.0) Assume that two compact connected and simple Lie groups $H$ and $H^{\prime}$ operate transitively and effectively on simply connected homological spheres of the same dimension $k$. If $H$ and $H^{\prime}$ are locally isomorphic, then there exists an isomorphism of $H$ onto $H^{\prime}$ carrying the isotropy subgroup of $H$ onto that of $H^{\prime}$; in particular the two homological spheres are homeomorphic.

Before the proof we recall the representation theory of Lie algebras.

Let $G$ be a compact connected simply connected semi-simple Lie group of rank $r$. Given a basis of the Cartan subalgebra of the Lie algebra of $G$, one can determine the simple roots $\alpha_{i}$ and the fundamental weights $L_{i}(1 \leqq i \leqq r)$, which are elements of the dual space of the Cartan subalgebra. Then an irreducible representation $\rho: G \rightarrow G L(n, C)$ corresponds to a linear combination $L=\sum p_{i} L_{i}, p_{i}$ being non-negative integers, in a one-to-one way up to the equivalence by Cartan's theorem [13]. In the sequel we shall confound $\rho$ with $L$. Let $\operatorname{deg} L$ be the degree $n$ of $\rho$ and $\delta$ be the sum of all $L_{i}$ 's. Then Weyl's theorem ([28]. See [10] also.) states:

$$
\operatorname{deg} L=n=\prod_{0<\alpha} \frac{(L+\delta, \alpha)}{(\delta, \alpha)}
$$

where the product is taken over all positive roots and the parentheses mean the inner product;

$$
\left(L_{i}, 2 \alpha_{j}\right)=\left(\alpha_{j}, \alpha_{j}\right) \delta_{i j}, \quad 1 \leqq i, j \leqq r,
$$

where $\delta_{i j}$ is Kronecker's $\delta . \quad \alpha_{i}, L_{i}$ and these inner products were calculated by E. Cartan (See his Oeuvres complètes, I vol. 1). In particular we have $\operatorname{deg}\left(L+L^{\prime}\right)>\operatorname{deg} L$. Actually the definition of $\delta$ in (5.1) is different from the one in [28], but it is justified by the following proposition, 
(5.3) For a semi-simple Lie algebra, $2 \delta$ equals the sum of all positive roots.

This is known, but we prove it here. Let $2 \beta$ be the sum of all positive roots and $S_{i}$ the reflection with respect to $\alpha_{i}$. If $\alpha=\sum m_{j} \alpha_{j}$ is a positive root other than $\alpha_{i}, S_{i}(\alpha)$ is also positive, because we have

$$
S_{i}(\alpha)=\alpha-2\left(\alpha, \alpha_{i}\right) \alpha_{i} /\left(\alpha_{i}, \alpha_{i}\right)=\sum_{j \neq i} m_{j} \alpha_{j}+\left(m_{i}-2\left(\alpha, \alpha_{i}\right) /\left(\alpha_{i}, \alpha_{i}\right)\right) \alpha_{i}
$$

and some $m_{j}(j \neq i)$ is positive. It follows that

$$
S_{i}\left(2 \beta-\alpha_{i}\right)=2 \beta-\alpha_{i} .
$$

Hence we get $S_{i}(\beta)=\beta-\alpha_{i} / 2+S_{l}\left(\alpha_{i}\right) / 2$, whence $S_{i}(\beta)=\beta-\alpha_{i}$. From $S_{l}(\beta)$ $=\beta-2\left(\beta, \alpha_{i}\right) \alpha_{i} /\left(\alpha_{i}, \alpha_{i}\right)$ we deduce $2\left(\beta, \alpha_{i}\right)=\left(\alpha_{i}, \alpha_{i}\right)$, while we have $2\left(\delta, \alpha_{i}\right)$ $=\left(\alpha_{i}, \alpha_{i}\right)$ by $(5.2)$; therefore we obtain $\beta=\delta$ or (5.3).

(5.4) Let $G$ be $O(n)$ or $U(n)$, and $V$ and $W$ be subgroups of $G$. If the representations of $V$ and $W$ obtained by restricting the natural injection of $G$ into $G L(n, C)$ are (absolutely) irreducible and equivalent, then they are conjugate in $G$.

There exists an element $\mu$ in $G L(n, C)$ such that we have $\mu \alpha=\beta \mu$ for any $\alpha \in V$ and $\beta \in W$. Assume that $G=O(n)$. Then we get $\bar{\mu} \alpha=\beta \bar{\mu}, \bar{\mu}$ being the complex conjugate of $\mu$. $\pi \mu^{-1}$ commutes with any $\alpha$ in $H$. By Schur's lemma, $\bar{\mu}$ is $\mu$ multiplied by a complex number $a^{2}$ of unit modulus. Observing $a \mu$ instead of $\mu$, we can suppose that $\mu$ is real. In the same way it is proved that the transposed matrix ${ }^{t} \mu^{-1}$ is $\mu$ multiplied by a positive number $b^{2}$. $b \mu$ belongs to $O(n)$ and we find that $b \mu V=W b \mu$. In case $G=U(n)$, it is verified in the same way that ${ }^{t} \bar{\mu}^{-1}$ is $\mu$ multiplied by a number $b^{2} . b^{2}$ is real and positive. Hence $b \mu$ belongs to $U(n)$ and $b \mu V=W b \mu$.

Now we begin the proof of (5.0). Let $K$ denote the isotropy subgroup of $H$. When $k=\operatorname{dim}(H / K)$ is even, the Euler characteristic of $H / K$ is positive. Since $H / K$ is simply connected, $K$ is connected. Hence Leray's theorem (4.11') applies; given $H$, the local structure of $K$ is determined. (The Betti numbers of compact simple groups are all known.) Thus in this case (5.0) follows from Wang's theorem (4.14).

Remark. Using the same method and (4.8), one can determine $H$ also. The pair $(H, K)$ is locally $\left(B_{r}, D_{r}\right), k=2 r$, or $\left(G_{2}, A_{2}\right), k=6$.

In case $k$ is odd, $K$ is not rationally homologous to zero in $H$ by Samelson's 
theorem (which follows directly from Theorem 18.4 in Koszul [14]). Hence the rational homology ring of $G$ is isomorphic to that of $(H / K) \times K$. By this fact combined with (4.7) and (4.8), the pair $(H, K)$ is locally $\left(D_{r}, B_{r-1}\right), 1 \leqq r$; $\left(A_{r-1}, A_{r-2}\right) ;\left(C_{r / 2}, C_{r / 2-1}\right), r=$ even; $\left(B_{4}, B_{3}\right), k=15 ;$ or $\left(B_{3}, G_{2}\right), k=7$; where we have put $k=2 r-1$.

The case $\left(D_{r}, B_{r-1}\right)$ can easily be treated with the method applied for other cases in the sequel or with the method of differential geometry. The details are omitted. To study other cases, we assume that $H$ is simply connected and prove that $H$ is effective. Then $K$ is simply connected, because we have $\pi_{2}(H / K)=0$ by $k>2$.

Case I: $\left.(H, K)=A_{r}, A_{r-1}\right)$. For the Lie algebra of $A_{m}$ we deduce from (5.1)

$$
\operatorname{deg} L_{k}={ }_{m+1} C_{k}, \operatorname{deg} 2 L_{k}={ }_{m+2} C_{k} \cdot{ }_{m+1} C_{k} /(k+1), \operatorname{deg}\left(L_{1}+L_{m}\right)={ }_{m+1} C_{2} .
$$

Let $\rho$ be the natural isomorphism of $H$ onto $S U(r+1)$. Restricting $\rho$ to $K$ we obtain a representation of degree $r+1$. In case $2<r$, an irreducible representation of $K$ with degree $\leqq r+1$ is of degree $r$ by the above and (5.4). Hence $\rho(K)$ fixes a direction. Since $S U(r+1)$ is transitive on the unit sphere, it follows that $\rho(K)$ is conjugate to $I+S U(r)$ in $S U(r+1)$, where $I+S U(r)$ is the set of all special unitary matrices with the $(1, j)$-elements equal to $\delta_{1} j$. Also in the sequel, the sign + between two matrix groups will denote their direct product in the diagonal block form, i.e. + means the notation of Hurwitz $\dot{+}$. Note that the identity group $I$ in the above cannot be replaced by a group other than $I$, for $K$ is simple and connected. In case $2=r$, if $\rho(K)$ is irreducible, $\rho$ is not faithful, contrary to the assumption of $\rho$. Hence $\rho(K)$ fixes a direction and the situation is analogous to the above. If $r=1, \rho(K)$ reduces to the identity. Since $\rho$ is an isomorphism onto, the proof for Case I is completed if $H$ is shown to be effective. If $N$ is a normal subgroup of $H$ contained in $K, \rho(N)$ is contained in the center of the simple group $\rho(H)=S U(r+1)$. Since $\rho$ is absolutely irreducible the subgroup $\rho(N)$ consists of matrices of diagonal form by Schur's lemma. On the other hand $\rho(N)$ is contained in $\rho(K)$ conjugate to $I+S U(r)$. Therefore $\rho(N)$ is trivial and $G$ is effective.

Case II: $(H, K)=\left(B_{4}, B_{3}\right)$. For $B_{m}$ we have $\operatorname{deg} L_{k}={ }_{2 m+1} C_{k}, k<m$, $\operatorname{deg} L_{m}=2^{m}$ and $\operatorname{deg} 2 L_{1}=m(2 m+3)$. In case $m=3$, the degrees of $L_{1}, L_{2}$, $L_{3}$, and $2 L_{1}$, are respectively $7,21,8$, and 27 . Moreover we need the facts; 
$\operatorname{deg}\left(L_{1}+L_{3}\right)=64$ and $\operatorname{deg} 2 L_{3}=40$. Let $\rho$ be the natural homomorphism of $H$ onto $S O(9)$. The kernel of $\rho$ is $Z_{2} . \quad \rho(K)$ admits an invariant space of dimension 7 or 8 . But the former is impossible, for otherwise $\rho(K)$ leaves fixed two real independent vectors and so is conjugate to $I+S O(7)$ in $S O(9)$, as is easily seen, whence $H / K$ must be the Stiefel manifold $\rho(H) / \rho(K)=S O(9) /(I+S O(7))$ $=V_{9,2}$. On the other hand we know $\pi_{i}\left(V_{9,2}\right)=Z_{2}\left([24]\right.$ p. 132), while $\pi_{7}(H / K)$ $=\pi_{i}\left(S^{15}\right)=0$, which leads to an obvious contradiction. Hence $\rho$ is the spin (therefore faithful) representation of $K$, if the operations of $\rho(K)$ are restricted to the 8-dimensional invariant space. Now (5.0) in Case II follows from (5.5) and the simple connectivity of $H$ (and $H^{\prime}$ ), if we show that $H$ is effective. Let $N$ be a normal subgroup of $H$ contained in $K . \rho(N)$ is then isomorphic to $N$ and contained in the center of $\rho(H)=S O(9)$. Hence $N$ is trivial.

Case III : $(H, K)=\left(B_{3}, G_{2}\right)$. For $G_{2}$ the degrees of $L_{1}, L_{2}$ and $2 L_{2}$ are 14 , 7 and 27 respectively. Let $\rho$ be the natural homomorphism of $G$ onto $S O(7)$. The representation of $K$ induced from $\rho$ must be unique up to an equivalence because of the above fact. On account of (5.5) it follows that any subgroup of $H$ isomorphic to $K$ is carried to $K$ by an automorphism of $H$. $H$ is effective on $S$, because the adjoint group of class $G_{2}$ is known to be simply connected and so $K$ cannot contain a discrete normal subgroup.

Case IV : $(H, K)=\left(C_{r}, C_{r-1}\right)(2<r)$. Let $\rho$ be the natural injection of $H$ into $S U(2 r) . \rho(H)$ is characterized in $S U(2 r)$ by the property: $\rho(G)$ leaves invariant a regular 2 -form $J$ ([8] p. 22). For $C_{r-1}$, we have $\operatorname{deg} 2 L_{1}=2 r^{2}+r-1$ and $\operatorname{deg} L_{h}={ }_{2 r} C_{h} \cdot(r-h) / r(1 \leqq h<r)$. In case $3<r$, the irreducible representation of $K$ obtained from $\rho$ is therefore of degree $2 r-2=\operatorname{deg} L_{1}$. Hence $\rho(K)$ is conjugate to $I+S p(r-1)$ in $S U(2 r)$. This holds in $S p(r)=\rho(H)$ as one sees from the facts that $S p(r)$ is transitive on the unit sphere, and that a transformation in $S p(r)$ which fixes a non-zero vector $x$, fixes the vector complex conjugate (with respect to some real base) to $J(x)$, which is linearly independent of $x$. The effectiveness of $H$ is proved as in Case I. In case $r=3$, the degrees of $L_{1}, L_{2}, L_{1}+L_{2}, 2 L_{2}$ are $4,5,16,10$ and 14 respectively. But $L_{2}$ is not the case, for otherwise $\rho(K)$ would fix only one direction, contrary to the above fact. Hence the proof is the same as in the case $3<r$.

Case V: $(H, K)=\left(C_{2}, C_{1}\right)$. Let $\rho$ be the natural projection of $H$ onto $S O(5) ; \rho$ is a local isomorphism with kernel $Z_{2} . \rho(K)$ is thus isomorphic either 
to $S p(1)$ or $S O(3)$. If $\rho(K)$ is isomorphic to $K=S p(1)$, then $\rho$, restricted to $K$, admits an irreducible invariant subspace of dimension 4 and $\rho(K)$ is conjugate to $I+S p(1)$. If $\rho(K)$ is isomorphic to $S O(3), K$ contains the kernel of $\rho$. Hence $S O(5) / \rho(K)$ must be homeomorphic to the 7 -sphere, contrary to the following proposition.

(5.5) (A. Hattori) Let $K$ be any subgroup of $S O(r), 4<r$, which is isomorphic with $\mathrm{SO}(3)$. Then the homogeneous space $B=S O(r) / K$ is not a simply connected homology sphere.

Assume the contrary. Since then $\pi_{1}(B)=0$ and the $i$-th cohomology group (over $\left.Z_{2}\right) H^{i}\left(B, Z_{2}\right)=0,0<i<3$, we have an exact sequence of Serre (Proposition 5 p. $468[23])$;

$$
H^{3}\left(B, Z_{2}\right) \rightarrow H^{3}\left(S O(r), Z_{2}\right) \rightarrow H^{3}\left(K, Z_{2}\right) .
$$

By Proposition 23.1 in Borel [2] this sequence reads

$$
H^{3}\left(B, Z_{2}\right) \rightarrow Z_{2}+Z_{2} \rightarrow Z_{2} \quad \text { (exact). }
$$

Hence $H^{3}\left(B, Z_{2}\right)$ cannot vanish, contrary to the assumption.

Remark. It is known that $B$ is a homological sphere over the rationals. Hence, in case $\pi_{1}(B)=0$, the 3 rd torsion group of $B$ is not trivial.

We have just accomplished the proof of (5.0), a special case of Theorem 2 . Assuming Theorem 2 to be true, we have corollaries to it.

Corollary 2.1. A simply connected homological sphere $S$ is homeomorphic and diffeomorphic to a sphere with the usual differentiable structure, if $S$ is a homogeneous space.

COROLlary 2.2. If a compact connected and simple Lie group $G$ is transitive on the $(n-1)$-sphere, then $G$ is isomorphic to $S O(n)$ or simply connected.

Let $N$ be the subgroup of $G$ consisting of the identity transformations. Then $G^{\prime}=G / N$ operates effectively and transitively on $S$. By $(5.0), G^{\prime}$ is simply connected or is isomorphic to $S O(n)$. Since $N=\pi_{1}\left(G^{\prime}\right)$, we obtain Corollary 2.2.

Corollary 2.3. If a compact connected Lie group $L$ operates transitively on a sphere $S$, then there exists on $S$ an L-invariant Riemannian metric of constant curvature.

This will be used in 6 before the complete proof of Theorem 2, There, 
however, $L$ will be simple, and in this case Corollary 2.3 follows from (5.0).

For later use we shall establish a lemma:

(5.6) Let $G$ be a compact connected Lie group. Let $\rho$ be a real orthogonal faithful representation of $G$ of degree $n$. If $\rho(G)$ is transitive on the unit sphere $S$, then $\rho$ is unique (up to an equivalence).

This was proved by H. Freudenthal [11]. But we give a proof for the sake of completeness. There exists a simple closed connected normal subgroup $H$ of $G$ such that $\rho(H)$ is transitive on $S$. Unless $H$ is $A_{n / 2-1}$ or $C_{n / 4}, \rho \mid H$ is absolutely irreducible and unique by (5.5) and the proof of (5.0). (The case $H=S O(2 n)$ can be treated directly or in the same way as other cases.) In particular we have $G=H$, and (5.6) is proved in this case. If $\rho$ is not absolutely irreducible, then $\rho$ is the real representation corresponding to an irreducible complex representation $\hat{\rho}$ of degree $n / 2$. Assume $H=A_{n / 2-1}$. The center of $H$ is of order $n / 2$. Since $\rho$ is faithful, this gives that $\rho$ is not absolutely irreducible. The complex representation $\hat{\rho}$ is $L_{1}$ or $L_{n / 2}$. But these two representations are complex conjugate to each other. Hence $\rho \mid H$ is unique. We have $\operatorname{dim} G / H \leqq 1$ and it is easy to see that $\rho$ is unique. In case $H=C_{n / 2}$, non-trivial complex representation of $H$ of degree $\leqq n$ is unique (of degree $n / 2$ ). The centralizer of $\rho(H)$ in $O(n)$ is isomorphic to $S p(1)$. Now $\rho$ is unique because of the fact that any one-parameter subgroup of $S p$ (1) can be transformed into any other one-parameter subgroup by some inner automorphism.

\section{Homogeneous sphere bundles}

This section is devoted to the proof of

Theorem 3. Let $S$ be a homogeneous $k$-sphere bundle whose bundle space, $S$ also, is an $(n-1)$-sphere. Then the principal bundle is $(G, H), F=G / H$, where we are in one of the following cases: (1) $G=H, F$ is a point, $k=n-1$, (2) $G=\operatorname{Spin}(9), H=\operatorname{Spin}(8), \quad F$ is the 8-sphere, $k=7=n-9, \quad(3) \quad G=\operatorname{Sp}(n / 4)$, $H=S p(n / 4-1) \times S p(1), F$ is the $(n-4)$-dimensional quaternion projective space, $k=3$, (4) $G=S U(n / 2), H=S U(n / 2-1) \times U(1), F$ is the $(n-2)$-dimensional complex projective space, $k=1$, and (5) $S$ is a double covering space of $F . F$ is the ( $n-1)$-dimensional real projective space, $k=0$. In cases (3) and (4) the structure group $H$ operates on the fibre $S^{k}$ as $S p(1)$ and $U(1)$ respectively.

Before the proof we note that, in the cases (2), (3) and (4), $H$ is not 
uniquely determined as a subgroup by the given conditions and yet the principal bundle is unique as shown in the proof and that in all cases the operations of the structure groups are unique by Theorem 2 or (5.0). Thus each condition about $(G, F, H)$ stated in Theorem 3 determine the bundle uniquely up to the equivalence.

The proof of Theorem 3. First the case $0=\operatorname{dim} F$ is evident. We assume $0<\operatorname{dim} F<n-1, F$ being the base space. $S$ is then simply connected. Hence there exists a simple compact and connected automorphism group of $S$ operating transitively, $G$, by the remark at the beginning of Section 4 . Let $H$ be the subgroup of $G$ leaving a fibre $S^{k}$ invariant. Then $F$ is $G / H$ and $S$ is associated with $(G, F, H)$. The local structures of $G$ and $H$ are given by (4.16). By Wang's theorem (4.14), the local "position" of $H$ in $G$ is also determined. By Corollary 2.2, $G$ is simply connected. Thus the pair $(G, H)$ is determined, and we obtain (2), (3) and (4). Then $H$ is simple or locally the direct product of two simple subgroups. Except the case $n=8$ in (4) (i.e. $H=S p(1) \times S p(1)$ ), it is obvious that $H$ acts on $S^{k}$ as one of the simple groups. The operation of $H$ on $S^{k}$ is thus uniquely determined by the local structure mentioned in the theorem. In the exceptional case, if $H$ does not act on the fibre $S^{3}$ as $S p(1), H$ does as $S O(4)$. Then, the bundle is the unit tangent bundle of $S^{4}=F$ as is easily seen. But the bundle space is known to be the Stiefel manifold $V_{5,2}$, which is not homeomorphic to a shpere because of the different homotopy types. Thus $H$ acts on $S^{3}$ as $S p(1)$ and the operation is uniquely determined. The only remaining case is now $\operatorname{dim} F=n-1$. $S$ is a double covering space of $F . F$ is a real projective space due to the following proposition.

(6.1) Any homogeneous space $F$ with a sphere $S$ as a double covering space is diffeomorphic to the real projective space.

There exists a compact connected and simple Lie group $G$ which is transitive and effective on $S$ and whose arbitrary element $\tau$ induces a transformation $\hat{\tau}$ on $F$ such that $\pi \tau=\hat{\tau} \pi, \pi$ being the projection of $S$ onto $F$. Since $G$ is simple, we can make use of Corollary 2.3, i.e. $S$ admits a $G$-invariant Riemannian metric of constant curvature. Denote by $\alpha$ the map of $S$ onto itself such that $\alpha(p)$ is the antipodal point of $p$ for any $p$ in $S$, "antipodal" ( = conjugate) being with respect to the above metric. Identifying $\alpha(p)$ with $p$ we obtain the real projective space $P$. Let $\phi$ be the covering transformation, i.e. $\phi$ is another involutive 
diffeomorphism of $S$ onto itself such that $\phi(p) \neq p$ and $\pi \phi(p)=\pi(p)$ for any $p$ in $S$. Both $\alpha$ and $\phi$ commute with any element in $G$. We shall prove $\alpha=\phi$, though this is evident if one uses (7.1) or Theorem 2. Suppose $\alpha \neq \phi$. Then $\phi(p) \neq \alpha(p)$ for any $p$ in $S$. Hence we can define a third map of $S$ onto $S$ such that $\delta(p)$ is the middle point of the shortest geodesic (which is well-defined) $\gamma(\phi(p), \alpha(p))$ joining $\phi(p)$ to $\alpha(p)$. Then $\delta \phi(p)$ is the middle point of $r(\phi \phi(p)$, $\alpha \phi(p))=\gamma(p, \alpha \phi(p))=\gamma(\alpha \alpha(p), \alpha \phi(p))$ which is $\alpha \gamma(\alpha(p), \phi(p))$ because $\alpha$ is an isometry. Hence we have $\delta \phi(p)=\alpha \phi(p)$ for any $p$. $\pi^{\prime}$ denoting the projection of $S$ onto $P$, it follows that $\pi^{\prime} \delta \pi^{-1}$ is a well defined map of $F$ into $P$. Since $\delta$ commutes clearly with any element in $G, \delta$ is a diffeomorphism of $S$ onto itself. Hence $\pi^{\prime} \delta \pi^{-1}$ is a diffeomorphism of $F$ onto $P$.

\section{Determination of $M$}

We will verify the first half of Theorem 1 , i.e. the existence of the diffeomorphism $\delta$. Because of (2.1) we may assume that $M$ is compact. We recall (2.4) and (1.6) where we put $N=M-\{p\}$ and $G$ is the group mentioned in (4.1); the differentiable bundle $M-\{p\}$ is determined uniquely by the subbundle $S$. $S$ is equivalent to one of the bundles in Theorem 3 (See Section 6). Repeating these arguments for the manifolds mentioned in Theorem 1 , we find that for some of the manifolds, $M^{\prime}$, the sphere-bundle $S^{\prime}$ is equivalent to $S$. Hence $M-\{p\}$ is diffeomorphic to $M^{\prime}-\left\{p^{\prime}\right\}$. The diffeomorphism $\delta$ extends to $M$ if one puts $\delta(p)=p^{\prime} . \quad \delta$ is a homeomorphism of $M$ onto $M^{\prime}$ by the uniqueness of the Alexandroff compactification. We have to prove that $\delta$ is diffeomorphic at $p$. Any geodesic issuing from $p$ arrives at $F$, forming a perpendicular to $F$ (Section 1). In fact, $M$ and $F$ being compact, there exists a perpendicular to $F$ starting at $p$ and moreover $M$ is isotropic at $p$. The analogous holds for $M^{\prime}$. By (1.7) these perpendiculars are mapped by $\delta$ to perpendiculars to $F^{\prime}$ issuing from $p^{\prime}$. We may assume that the distance $d(p, F)$ equals $d^{\prime}\left(p^{\prime}, F^{\prime}\right)$, and $\delta$ is an isometry if restricted to a perpendicular to $F$ issuing from $p$ therefore to any of such perpendiculars.

Now we define a mapping $\lambda$ of the tangent space $T$ to $M$ at $\phi$ into the tangent space $T^{\prime}$ to $M^{\prime}$ at $p^{\prime}$. For any $X$ in $T$, let $r$ be the geodesic with the initial tangent $X$. By definition $\lambda(X)$ is the initial tangent (of the same length as $X)$ of the geodesic $\delta(\gamma)$ starting at $p . \quad \lambda$ commutes with $G ; \tau \lambda(x)=\lambda \tau(X)$ for any $\tau \in G$ and any $X \in T$, where we have identified the connected simple 
group $G$ on $M$ with $G^{\prime}$ on $M^{\prime}$ (because they are isomorphic) and we have confounded $\tau$ with the one induced from $\tau$ on $T$ to $T^{\prime}$. Consider a normal coordinate system $\nu$ of $M$ with center $p . \quad \nu$ is a diffeomorphism of a neighborhood $U$ of $p$ in $M$ onto a neighborhond $V$ of $p$ in $T$. Let $\nu^{\prime}$ be an analogous for $M^{\prime}$. We assume that $U$ and $U^{\prime}$ are $G$-invariant and $\delta(U)$ is $U^{\prime}$. Restricted to $V, \lambda$ coincides with $\nu^{\prime} \circ \delta \circ \nu^{-1}$. If $\lambda$ is linear then $\delta$ is diffeomorphic at $p$. By (5.7) there exists a linear isomorphism $\lambda^{\prime}$ of $T$ onto $T^{\prime}$ which commutes with $G$. $\lambda^{-1} \lambda^{\prime}$ is thus a length-preserving map of $T$ onto $\mathrm{T}^{\prime}$ which commutes with $G$ and with the multiplication by a positive number. This (therefore $\lambda$ ) is linear if the following proposition (7.1) is true; i.e. the existence of $\delta$ follows from (7.1).

(7.1) Let $T$ be an $n$-dimensional real vector space with a euclidean metric, and $G$ be a subgroup of the orthogonal group $O(T)$ of $T$ which is transitive on the unit $(n-1)$-sphere $S$. Assume that $\mu$ is a homeomorphism of $T$ onto itself such that

1) $\mu$ leaves invariant $S$,

2) $\mu(c X)=c \mu(X)$ for any positive number $c$ and any $X$ in $T$,

3) $\mu$ commutes with $G: \mu \circ \tau=\tau \circ \mu$ for each $\tau$ in $G$.

Then $\mu$ is linear.

(This is a special case of Theorem 2.)

Under this hypothesis, we assume without loss of generality that $G$ is compact connected and simple. We need some lemmas.

(7.2) If $\mu(X)$ and $X$ are linearly dependent for some unit vector $X$ in $T$, then $\mu$ is linear.

In fact $\mu(X)$ is then equals $e X$ where $e=+1$ or -1 . For any unit vector $Y$ there exists some $\tau$ in $G$ carrying $X$ to $Y$. We have

$$
\mu(Y)=\mu \tau(X)=\tau \mu(X)=\tau(e X)=e \tau(X)=e Y .
$$

Hence $\mu$ is linear.

(7.3) If a unit vector $X$ and $\mu(X)$ are linearly independent, then

a) $\mu(X)$ is left invariant by the group $K$ leaving $X$ fixed and

b) there exists a non-zero G-invariant continuous vector field on $S$ (which is a submanifold of $T$ with the usual differentiable manifold structure).

For each $\tau$ in $K, \tau \mu(X)=\mu \tau(X)=\mu(X)$, which implies a). Let $Y(\neq 0)$ be 
a linear combination of $X$ and $\mu(X)$ such that $Y$ is orthogonal to $X . \quad Y$ is invariant-under $K$ by a). Let $Y^{\prime}$ be a vector at the point $X$ which is parallel to $Y$. $Y^{\prime}$ is tangent to $S$ at the point $X$. Since $G$ is transitive on $S$, it follows that there exists a $G$-invariant vector field $y$ on $S$ assigning $Y^{\prime}$ to $X$. b) is thus proved.

(7.4) In case $G=S O(n)$, $\mu$ is linear.

Given a unit vector $X, K$ leaves fixed no vectors other than $\pm X$. Hence by (7.3) and (7.2) we get (7.4).

(7.5) If $n$ is odd, $\mu$ is linear.

Then the Euler characteristic of $S$ is not zero. Hence $X$ and $\mu(X)$ are linearly dependent by (7.3) b). By (7.2) we have (7.5).

To complete the demonstration of (7.1), it suffices to investigate the cases: $G=S U(n / 2), S p(n / 4), \operatorname{Spin}(7)(n=8)$ or $\operatorname{Spin}(9) \quad(n=16)$ owing to (4.7) and Corollary 1.2. The first two are the usual real representations and the last two are the spin representations.

(7.6) If $G$ is $S U(n / 2)$ or $S p(n / 4), \mu$ is linear.

We prove this only in the second case; the first is proved analogouly with more ease. Then, putting $n / 4=m, T$ can be regarded as an $m$-dimensional $Q$ right-module $Q^{m}$ with the symplectic product, $Q$ being the quaternion algebra [8], so that $G$ is the automorphism group of $Q^{m}$. For any unit vector, the subgroup $K$ fixing $X$ leaves invariant only the elements of the form $X q(q \in Q)$ Hence by (7.4) we have $\mu(X)=X q_{0}$ for some $q_{0}$. The left multiplication can also be defined on $Q^{m}$ and we can identify $q X$ with $X q$ for this fixed $X$ and any $q$ in $Q$. Now we define a mapping $\nu$ of $Q^{m}$ onto itself ; $\nu: Y \in Q^{m} \rightarrow q_{0} Y$. ${ }^{\prime}$ is not an automorphism of $Q^{m}$ but an automorphism of the real vector space $T$, and belongs to the orthogonal group $O(T)=O(n)$, because the norm of $q_{0}$ is unity. Further $\nu(X)=\mu(X)$ and $\nu$ commutes with any element of $G$. Thus $\nu$ coincides with $\mu$ on $T$; in particular $\mu$ is linear.

(7.7) If $G$ is $\operatorname{Spin}(7)$ or Stin(9), $\mu$ is linear.

It suffices to prove that $K$ leaves invariant just one independent vector. Assume that $G=\operatorname{Spin}(9)$. $\rho$ shall denote the spin representation of $G$ of degree 16. The weights are $\left( \pm \lambda_{1} \pm \lambda_{2} \pm \lambda_{3} \pm \lambda_{1}\right) / 2$. It follows that the multipicity of each weight equals one. $\rho(G)$ is a linear transformation group on the complexification 
of $T$, which will be denoted by $T$ also. Each vector $Y$ in $T$ is uniquely written as the sum of the eigenvectors $Y_{\alpha}$ belonging to the weights $L_{(\alpha)}, 1 \leqq \alpha \leqq 16$. If $Y$ is left invariant by $K$, then each $Y_{\alpha}$ is invariant by the Cartan subgroup of $K$. If further $Y_{\alpha}$ is different from zero, then $L_{(\alpha)}$ vanishes on the Cartan subalgebra of $K$. This occurs for at most two values of $\alpha$, because $L_{(\alpha)}$ is linearly independent of any $L_{(\beta)}$ other than $\pm L_{(\alpha)}$ and the difference of the dimensions of the Cartan subalgebras of $G$ and $K$ equals one. Assume that there exist two such vectors. Then the restriction of $\rho$ to $K$ is the direct sum of two representation; the one is trivial and of degree two, the other (of degree 14) admitting no invariant vectors. Since $K$ is $B_{3}$ (See Case II in the proof of (5.0), Section 5$), \rho^{\prime}$ is the direct sum of two equivalent irreducible representations $\rho^{\prime \prime}$ of degree 7. From the fact that $\rho^{\prime \prime}$ is not faithful, one easily finds that $\rho$ is not faithful, which leads to a contradiction. Thus $K$ leaves fixed just one independent vector.

In case $G=\operatorname{Spin}(7)$, if $K$ leaves invariant two vectors, $\mathrm{K}=G_{2}$ must admit a faithful representation of degree 6 , contrary to the fact shown in Case III in the proof of (5.0).

As a corollary to (7.1) we obtain another part of Theorem 2 .

(7.8) Under the assumption of Theorem 2, there exists a homeomorphism $\beta$ of $S$ onto $S^{n-1}$ which induces an isomorphism $\alpha$ of $H^{\circ} \cdot C$ into $O(n)$ carrying $H^{\circ}$ onto $H^{\prime}$.

Let $G$ be a simple closed connected normal subgroup of $H^{\circ}$ which is transitive on $S$. By (5.0) there exists a homeomorphism $\beta^{\prime}$ of $S$ onto $S^{n-1}$ which induces an isomorphism $\alpha^{\prime}$ of $G$ into $H^{\prime}$. $\quad \alpha^{\prime}$ extends to $H^{\circ} \cdot C$ in such a way that for each $\tau$ in $H^{\circ} \cdot C$ we have $\alpha^{\prime}(\tau)=\beta^{\prime} \tau \beta^{\prime-1}$, which is a topological transformation of $S^{n-1}$. For brevity we identify $S$ with $S^{n-1}$, and neglect $\alpha^{\prime}$ and $\beta^{\prime}$. $G$ becomes a subgroup of $H^{\prime}$. We imbed $S$ into the $n$-dimensional euclidean space $T$ in a natural way. Let $C_{G}$ be the centralizer of $G$ in the topological transformation group of $S$. For each $\tau$ in $C_{G}$ there exists a homeomorphism $\pi(\tau)=\mu$ of $T$ onto itself satisfying the conditions 1$), 2$ ) and 3) in (7.1). By (7.1), $\mu$ is linear. Therefore $C_{G}$ is contained in $O(n)=O(T)$. Since we have $H^{\circ} \cdot C \subset G \cdot C_{G}$, this gives that $H^{\circ} \cdot C$ is a subgroup of $O(n)$; i.e. $\alpha^{\prime}$ is a faithful representation of $H^{\circ}$. By (5.0), $H^{\circ}$ and $H^{\prime}$ are isomorphic. Hence by (5.7) there exists a homeomorphism $\beta^{\prime \prime}$ of $S^{n-1}$ onto itself which induces an 
automorphism of $H^{\circ}$ onto $H^{\prime}$. Now $\beta=\beta^{\prime \prime} \beta^{\prime}$ and $\alpha=\alpha^{\prime \prime} \alpha^{\prime}$ are the required mappings.

(7.9) (Cartan) Let $A(G)$ be the automorphism group of a Lie group, and $I(G)$ be the group of inner automorphisms of $G$. Then $A(G)$ coincides with $I(G)$ when $G=S p(m), S O(2 m-1)$, Spin(7), Spin(9), or the compact connected group $G_{2} . \quad A(G) / I(G)$ is $Z_{2}$ when $G=S U(m), 2<m$, or $S O(2 m)$. (Gantmacher [12]. See [11] also.)

When $G=S U(m), 2<m$, an outer automorphism of $G$ is obtained by assigning the complex conjugate to each element in $G$. When $G=S O(2 m)$, an outer automorphism is induced by the symmetry with respect to a hyperplane.

Using (7.1) again we will complete the proof of Theorem 2. For this purpose it is sufficient to show

(7.10) The homeomorphism $\beta$ in (7.8) induces an isomorphism of $H$ (in Theorem 2) into $O(n)$.

Each element $\tau$ in $H$ defines an automorphism $a d(\tau)$ of $G$ (defined in the proof of (7.8). Put $G=H^{\circ}$ when $\left.H^{\circ}=S O(4), n=4\right)$ which carries $\lambda \in G$ to $\tau \lambda \tau^{-1}$. Let $\alpha(\tau)$ denote the topological transformation $\beta \tau \beta^{-1}$. The automorphism $a d(\tau)$ of $G$ defines an automorphism of $\alpha(G)$, which may be written as $\operatorname{ad}(\alpha(\tau))$. By (7.9) there exists an element $\tau^{\prime}$ of $O(n)$ with $\operatorname{ad}(\alpha(\tau))=a d\left(\alpha\left(\tau^{\prime}\right)\right)$ on $G$. By (7.1), $\mu=\alpha(\tau)^{-1} \tau^{\prime}$ thus belongs to $O(n)$. Therefore we have $\alpha(H) \subset O(n)$. Obviously $\alpha$ is an isomorphism of $H$.

Now we verify the second half of Theorem 1 :

(7.11) Under the assumption of Theorem 1 , the diffeomorphism $\delta$, induses an isomorphism of $J$ into the isometric isotropy group $J^{\prime}$ of $M^{\prime}=\delta(M)$ at $p^{\prime}=\delta(p)$.

$\delta$ induces an isomorphism $\alpha$ of $J$ into the orthogonal group $O(n)$ of the tangent space $T^{\prime}$ at $p^{\prime}$. We have to show that $J^{\prime}$ contains $\alpha(J)$. This is clear when $M^{\prime}$ is the euclidean space, a sphere, or a real elliptic space, $J^{\prime}$ being then $O(n)$. Let $G$ denote a connected simple normal subgroup of $J$ which is transitive on the unit sphere. In order that $J^{\prime}$ contains $\alpha(J)$, it is sufficient that $J^{\prime}$ is the normalizer of $\alpha(G)$ in $O(n)$. In case $M^{\prime}$ is the complex elliptic space, $J^{\prime}$ has two connected components and the identity component is $U(n / 2)$, while $\alpha(G)=S U(n / 2)$. In case $M^{\prime}$ is the quaternion elliptic space, $J^{\prime}$ is connected and the local product of $\alpha(G)=S p(n / 4)$ and its centralizer $(=S p(1))$. In case 
$M^{\prime}$ is the Cayley projective plane, we have $J^{\prime}=\alpha(G)=\operatorname{Spin}(9)$. In all these cases $J^{\prime}$ is the normalizer of $\alpha(G)$ by (7.9).

We have just completed the proof of Theorem 1.

Corollary 1.1. If a compact and connected $C^{3}$-transformation group $G$ of a connected and paracompact differentiable manifold $M$ admits a fixed point $p$ and an $(n-1)$-dimensional orbit, then $M$ is $C^{1}$-diffeomorphic to one of the spaces mentioned in Theorem 1.

$M$ admits a $G$-invariant Riemannian metric of class $C^{2}$. We assume that $n$ is greater than 1. The condition (1.1) is satisfied if one puts $N=M-F$ where $F$ is the farthest points from $p$ if $M$ is compact and otherwise $F$ is an empty set. From (1.5) and the fact that $F$ is a rare set, we easily see that the hypothesis of Theorem 1 is satisfied.

A metric space $M$ is said two-point homogeneous if, given four points $a, b$, $a^{\prime}, b^{\prime}$ with the distance $d(a, b)=d\left(a^{\prime}, b^{\prime}\right)$, there exists an isometry of $M$ carrying a to $b$ and $b$ to $b^{\prime}$.

CoROLLARY 1.2. Under the hypothesis of Theorem 1, there exists on $M a$ Riemannian metric so that $M$ becomes a two-point homogeneous space.

Note that two-point homogeneity is enjoyed by all the spaces mentioned in Theorem 1, i.e. the euclidean space, the sphere, and the elliptic spaces.

Corollary 1.3 The hypothesis of Theorem 1 being preserved, the set of the conjugate points ( nearest to $p$ ) of $p$ is the empty set, one point $\neq p$, one-point $p$, an (n-2)-dimensional complex projective space, an $(n-4)$-dimensional quaternion projective space, or the 8-sphere, according as $M$ is diffeomorphic to the euclidean space, the sphere, the real projective space, the complex projective space, the quaternion projective space or the Cayley projective plane.

In fact $F$ is clearly the set, except the case $M$ is the real projective space. In this case the proof is easy and omitted.

Remark. In that exceptional case $F$ is the cut locus. Using Bott's result [6] or directly, one can readily calculate the number (counted with multiplicity) $k$ of the conjugate points of $p$ on a geodesic issuing from $p$. 


\section{The homogeneous case}

Let $M$ be an $n$-dimensional connected Riemannian space. Assume that a connected isometry group $I$ of $M$ is transitive, and $M$ is $J$-isotropic at $p$ where $J$ is the isotropy subgroup of $I$ at a point $p$ of $M$. By means of the results in Sections 1 to 3 , we shall determine $I, J$ and the $I$-invariant Riemannian metric on $M$.

The linear isotropy group is irreducible. It follows from an unpublished theorem of Matsushima (see the Appendix) that $M=I / J$ is a symmetric space unless $M$ is compact. The corresponding compact symmetric space is isotropic too.

We suppose that $M$ is compact. Consider the case $(A)$ where $M$ is diffeormorphic to the sphere or the real projective space. The universal covering Riemannian space $M$ which is diffeomorphic to the sphere (if $1<n$ ) is homogeneous and isotropic. By Corollary 2.3 there exists on $M$ an $I$-invariant Riemannian metric of constant curvature admitting the same isotropy group. Since the linear isotropy group is irreducible, this metric is the unique $I$-invariant one (up to a constant factor). Hence $M$ is either a (Riemannian) sphere or the real elliptic space.

Assume that we are not in the case $(A)$. Let $S$ and $F$ be as in Section 2 . $S$ divides $M$ into two parts. Let $X_{1}$ be the closure in $M$ of the connected component of $M-S$ containing $p$, and $X_{2}$, be the closure of the other component. Then we have $M=X_{1} \cup X_{2}$ and $S=X_{1} \cap X_{2} . \quad X_{1}$ is a closed cell. $\quad F$ is a deformation retract of $X_{2}$. Hence by (3.4) and the Mayer-Vietoris sequence [19], we obtain the Poincaré poly nomial of $M$ :

$$
P(t ; M)=P(t ; F)+t^{n}=1+t^{k+1}+\cdots+t^{(m+1)(k+1)} .
$$

In particular the Euler characteristic of $M$ is positive, and $I$ has the same rank as $J$. By Leray's theorem $\left(4.11^{\prime}\right)$ the Poincaré polynomial of $I$ is calculated from that of $J$. It follows from Theorem 3 that the pair $(I, J)$ is locally $\left(A_{n / 2}\right.$, $\left.A_{n / 2-1} \times T\right),\left(C_{n / 1+1}, C_{n / 4} \times C_{1}\right)$ or $\left(F_{4}, B_{1}\right)$, where $T$ is the one-dimensional toral group. (The pair $\left(B_{r}, C_{r-1} \times C_{1}\right), 2<r$, is impossible because $B_{r}$ does not contain such a group as is seen, e.g. from the fact that the least degrees of the locally faithful real representations of $B_{r}$ and $C_{r-1}$ are $2 r+1$ and $4 r-4$ respectively.

Since $M$ is simply connected by (2.5) and (3.1), these local structures 
determine the global structures of $I, J$ and $M$ by Wang's theorem (4.14). The invariant Riemannian metric on $M$ is unique up to a constant factor, and we conclude that $M$ is the complex or quaternion elliptic space or the Cayley elliptic plane.

Remark 1. The group of all the isometries of $M$ has a finite number of connected components as is clear from the above. It was determined by Freudenthal [11].

Remark 2. There exist on $M$ many other $I$-invariant metrics which are not Riemannian (Wang [26]).

\section{Appendix}

We prove the following theorem of Matsushima :

Theorem. Let $M=G / K$ be a Riemannian homogeneous space, $G$ being the maximal connected isometry group. Assume that 1) $M$ is not compact and 2) the linear isotropy group is irreducible. Then $M$ is a symmetric space. (The proof is analogous to that of the complex case [16].)

$K$ is compact by Dantzig-Waerden's theorem. From 1) and 2), it follows that $K$ is a maximal compact subgroup of $C$; in particular $K$ is connected. $M$ is homeomorphic to the euclidean space by Malcev-Iwasawa's theorem. If $G$ is semi-simple, $M$ is a symmetric space by Cartan's theorem. Suppose that $G$ is not semi-simple, i.e. that the radical $N$ is not discrete. $N$ contains a nondiscrete connected normal abelian subgroup $A$ of $G$. Since $G$ is effective, $K$ does not contain $A$. By 2), $A$ is transitive on $M$. Therefore the invariant metric is locally euclidean. $M$ is thus isometric to the euclidean space, which is a symmetric space.

\section{BIBLIOGRAPHY}

[1] A. Borel, Le plan projectif des octaves et les sphères comme espaces homogènes. C. R. Paris, 230 (1950), 1378-1380.

[2] A. Borel, Sur la cohomologie des espaces fibrés principaux et des espaces homogènes de groupes de Lie compacts, Ann. of Math., 57 (1953), 115-207.

[3] A. Borel, Les bouts des espaces homogènes de groupes de Lie, Ann. of Math., 58 (1953), 443-457.

[4] A. Borel, Topology of Lie groups and characteristic classes, Bull. Amer. Math. Soc., 6 (1955), 397-432. 
[ 5 ] R. Bott, The stable homotopy of the classical groups, Proc. Nat. Acad. Sci. U.S.A., 43 (1957), 933-935.

[6] R. Bott, On manifolds all of whose geodesics are closed, Ann. of Math., 60 (1954), 375-382.

[ 7 ] S. S. Chern, Topics in differential geometry, Princeton, 1951.

[8] C. Chevalley, Theory of Lie groups I. Princeton, 1946.

[9] S. Eilenberg and N. Steenrod, Foundations of algebraic topology, Princeton, 1952.

[10] H. Freudenthal, Zur Berechnung der Charaktere der halb-einfachen Lieschen Gruppen, I. Indag. Math., 16 (1954), 369-376.

[11] H. Freudenthal, Neuere Fassungen des Riemann-Helmholtz-Lieschen Raumproblems, Math. Z., 63 (1956), 374-405.

[12] F. Gantmacher, Canonical representation of automorphisms of a complex semi-simple Lie group, Math. Sbornik., 5 (1939), 101-144.

[13] Harisch-Chandra, On some applications of the universal enveloping algebra of a semi-simple Lie algebra, Trans. Amer. Math. Soc., 70 (1951), 28-96.

[14] J. L. Koszul, Homologie et cohomologie des algèbres de Lie, Bull. Soc. Math. France, 78 (1950), 65-127.

[15] J. L. Leray, L'homologie d'un espace fibré dont la fibre est connexe, J. Math. Pures Appl., 29 (1950), 169-213.

[16] Y. Matsushima, Un théorème sur les espaces homogènes complexes, C. R. Paris., 241 (1955), 785-787.

[17] Y. Matsushima, On a type of subgroups of a compact Lie group, Nagoya Math. J, 2 (1951), 1-51.

[18] D. Montgomery and H. Samelson, Transformation groups of spheres, Ann. of Math., 44 (1943), 454-470.

[19] D. Montgomery and L. Zippin, Topological transformation groups, New-York, 1955.

[20] P. S. Mostert, On a compact Lie group acting on a manifold, Ann. of Math., 65 (19.57), $447-455 ; 66$ (1957), p. 589.

[21] T. Nagano, Transformation groups with $(\boldsymbol{n}-\mathbf{1})$-dimensional orbits, Nagoya Math. J., 14 (1959), 25-38.

[22] H. Samelson, Topology of Lie groups, Bull. Amer. Math. Soc., 58 (1952), 2-37.

[23] J. P. Serre, Homologie singulière des espaces fibrés. Applications, Ann. of Math., 54 (1951), 425-505.

[24] N. Steenrod, The topology of fibre bundles, Princeton, 1951.

[25] J. Tits, Sur certaines classes d'espaces homogènes de groupes de Lie, Bruxelles, 1955.

[26] H. C. Wang, Two-point homogeneous spaces, Ann. of Math., 55 (1952), 177-191.

[27] H. C. Wang, Homogeneous spaces with non-vanishing Euler characteristics, Ann. of Math., 50 (1949), 925-953.

[28] H. Weyl, Theorie der Darstellung knotinuierlicher halb-einfacher Gruppen durch lineare Transformationen, III. Math. Z. 24 (1926), 377-395.

\section{Mathematical Institute \\ College of General Education}

University of Tokyo 